\title{
Enfrentar las desigualdades en salud en América Latina: el rol de la protección social
}

\author{
Addressing health inequalities in Latin America: the role of social \\ protection
}

Laís Abramo (https://orcid.org/0000-0002-3385-4446) ${ }^{1}$

Simone Cecchini (https://orcid.org/0000-0002-9757-3141) ${ }^{1}$

Heidi Ullmann (https://orcid.org/0000-0002-3730-9509) ${ }^{1}$

${ }^{1}$ División de Desarrollo Social, CEPAL. Av. Dag Hammarskjöld 3477,

Vitacura. Santiago de

Chile Chile.

simone.cecchini@cepal.org

\begin{abstract}
After more than a decade of progress in various areas of social development, since 2015 poverty has increased, labor market indicators have deteriorated, and the reduction of income inequality has stagnated in Latin America. These trends are of concern as they can affect health indicators and exacerbate profound health inequalities. This situation demands integrated policy responses that can create synergies between different sectors. There is growing recognition of the role of social protection in the eradication of poverty and the reduction of inequality. Various social protection mechanisms buffer against the costs of accessing health services directly and indirectly. By expanding coverage and universal access, promotion and prevention actions in health and nutrition, and fundamentally, the fight against poverty, inequality, and exclusion, social protection plays a fundamental role in guaranteeing the right to health and overcoming inequalities in this area. The reduction of inequalities in health should be a priority for all countries, and a way forward in that direction is to promote the construction and strengthening of universal social protection systems.
\end{abstract}

Key words Inequality, Health, Social protection, Latin America
Resumen Después de más de una década de avances en diversos ámbitos del desarrollo social, desde 2015 en América Latina se registran aumentos en la pobreza, deterioros en indicadores del mercado laboral y un estancamiento en la reducción de la desigualdad del ingreso. Estas tendencias son preocupantes ya que pueden perjudicar los indicadores de salud y exacerbar las profundas desigualdades en salud. Esta situación demanda respuestas integradas de politicas, capaces de crear sinergias entre diferentes sectores. Existe un creciente reconocimiento sobre el rol de la protección social en la erradicación de la pobreza y la reducción de la desigualdad. Los diversos mecanismos de protección social aminoran los costos de acudir a servicios de salud de manera directa e indirecta. Mediante la expansión de la cobertura $y$ el acceso universal, las acciones de promoción y prevención en salud y nutrición, $y$ de manera fundamental, la lucha contra la pobreza, la desigualdad y la exclusión, la protección social juega un papel ineludible para la garantía del derecho a la salud y la superación de desigualdades en esta área. La reducción de las desigualdades en salud debe ser una prioridad para todos los países y un camino para avanzar en esa dirección es promover la construcción y el fortalecimiento de sistemas de protección social universales.

Palabras clave Desigualdad, Salud, Protección social, América Latina 


\section{Introducción}

La desigualdad, en sus múltiples expresiones, es un problema estructural de gran envergadura en América Latina. Después de más de una década de avances en diversos ámbitos del desarrollo social en la región, entre los cuales se destacan la reducción de la pobreza, de la desigualdad de ingresos y el aumento de la inclusión social y laboral, desde 2015 se registra un aumento en la pobreza, en particular la pobreza extrema, el aumento del desempleo, el deterioro en indicadores de la calidad del empleo y el estancamiento en la reducción de la desigualdad del ingreso. Estas tendencias son preocupantes también desde la perspectiva de las desigualdades en salud, ya que pueden perjudicar, de manera directa o indirecta, los indicadores de salud y nutrición y exacerbar las profundas brechas existentes en esta área. A su vez, los retrocesos en los indicadores de salud y una expansión de las disparidades en salud contribuyen a propagar un ciclo vicioso de mal estado de salud, pobreza y desigualdad. En este escenario es imperativo fortalecer y ampliar los sistemas de protección social y promover políticas redistributivas para mitigar los impactos negativos de ese proceso sobre el bienestar de la población y desarrollar medidas que promuevan su plena inclusión social y laboral y el pleno derecho a la salud. El presente artículo tiene por objetivo mostrar evidencias sobre las desigualdades en salud en América Latina y su evolución reciente y reflexionar sobre el rol de la protección social para reducir esas desigualdades.

\section{Evolución reciente de la pobreza y la desigualdad del ingreso en América Latina}

La erradicación de la pobreza y la pobreza extrema, así como la reducción de la desigualdad, en todas sus manifestaciones, son grandes retos para los países de América Latina. Entre 2002 y 2014 la región logró notables avances en estas áreas y en otros indicadores sociales, como en el ámbito de la educación y la salud. Los logros en la reducción de la pobreza, la pobreza extrema y la desigualdad de ingresos en ese período estuvieron asociados a la evolución positiva del mercado de trabajo (en particular a la disminución del desempleo, a un aumento en la formalización del trabajo y mejorías en los ingresos laborales) y a la expansión y fortalecimiento de los sistemas de protección social. A partir del 2015 se registran retrocesos: las tasas de pobreza y extrema pobreza aumen$\tan$ respectivamente de $27,8 \%$ y $7,8 \%$ en 2014 a $30,2 \%$ y $10,2 \%$ en $2017^{1}$.
Este escenario, que ocurre en un contexto de débil crecimiento económico y restricciones fiscales, con aumentos en las tasas de desempleo y de la informalidad laboral, enciende señales de alerta por los impactos negativos que puede tener a corto y largo plazo sobre el bienestar de la población ${ }^{2}$. Asimismo, se observan significativas desigualdades en la incidencia de la pobreza y pobreza extrema según el sexo, la edad, la condición étnico-racial y el área de residencia de las personas. Ella es más aguda en las personas que viven en el ámbito rural, los niños, niñas, adolescentes y jóvenes, los pueblos indígenas, la población afrodescendiente, las mujeres en edad productiva, $y$ las personas con menores niveles de educación ${ }^{1}$. Las poblaciones rurales y los niños, niñas, adolescentes y jóvenes registraron además aumentos en la incidencia de la pobreza entre 2012 y $2017^{1}$.

La desigualdad de ingresos disminuye de forma significativa entre 2002 y 2017 (el índice de Gini se reduce del 0,53 al 0,47). Sin embargo, el ritmo de esa reducción se enlentece entre 2014 y 2017 y persisten altos niveles de desigualdad, posicionando a América Latina como la región más desigual del mundo ${ }^{1}$. La reducción de las brechas entre los grupos de menores y mayores recursos se debió en gran medida a la mejoría de los ingresos laborales en los estratos de menores ingresos. Además, las pensiones y transferencias jugaron un papel importante, particularmente en los estratos de menores ingresos ${ }^{1}$.

\section{Más allá de la desigualdad de ingresos}

La desigualdad es una característica histórica y estructural de las sociedades latinoamericanas que incluye la desigualdad económica o de medios (ingresos, propiedades, activos financieros y productivos), la desigualdad en el goce de los derechos, en el desarrollo de capacidades, en el acceso a oportunidades, a la autonomía y al reconocimiento recíproco ${ }^{3}$. El concepto de la "matriz de la desigualdad social" (Cuadro 1) contribuye a avanzar el análisis y la reflexión de este fenómeno complejo, multidimensional y multicausal con miras a orientar políticas para su reducción. Una de las contribuciones de este concepto es que propone un abordaje de la desigualdad que contempla la confluencia de múltiples y simultáneas formas de discriminación y exclusión y la manera en que estas derivan en desigualdades en salud y en otros ámbitos del desarrollo social, que a su vez se retroalimentan entre sí.

La matriz de la desigualdad social está condicionada por la matriz o estructura productiva 
Cuadro 1. Matriz de la desigualdad social.

\begin{tabular}{|c|c|c|}
\hline Planteamientos teóricos & Ejes estructurantes & $\begin{array}{c}\text { Ámbitos de derechos en que } \\
\text { inciden }\end{array}$ \\
\hline $\begin{array}{l}\text { Heterogeneidad estructural } \\
\text { (matriz productiva) } \\
\text {. Cultura del privilegio } \\
\text {. Concepto de igualdad: } \\
\text { - Igualdad de medios (ingresos y } \\
\text { recursos productivos) } \\
\text { - Igualdad de derechos } \\
\text { - Igualdad de capacidades } \\
\text { - Autonomía y reconocimiento } \\
\text { recíproco }\end{array}$ & $\begin{array}{l}\text { Nivel socioeconómico } \\
\text { Género } \\
\text {. Raza y etnia } \\
\text {. Etapa del ciclo de vida } \\
\text { Territorio } \\
\text {. Situación de discapacidad } \\
\text {. Estatus migratorio } \\
\text {. Orientación sexual e identidad de } \\
\text { género }\end{array}$ & $\begin{array}{l}\text {. Ingresos } \\
\text {. Trabajo y empleo } \\
\text {. Protección social y cuidados } \\
\text {. Educación } \\
\text {. Salud y nutrición } \\
\text {. Servicios básicos (agua, } \\
\text { saneamiento, electricidad, } \\
\text { vivienda, TIC) } \\
\text {. Seguridad ciudadana y vida libre } \\
\text { de violencia } \\
\text {. Participación y toma de } \\
\text { decisiones }\end{array}$ \\
\hline
\end{tabular}

Fuente: Comisión Económica para América Latina y el Caribe (CEPAL), Hacia una agenda regional de desarrollo social inclusivo: bases y propuesta inicial (LC/MDS.2/2), Santiago, $2018^{4}$.

de la región, que concentra el empleo en trabajos informales y de baja calidad, con bajos ingresos y limitado o nulo acceso a mecanismos de protección social. El mercado laboral es el eslabón que vincula a una estructura productiva heterogénea (e inherentemente desigual en términos de productividad, acceso y calidad de los empleos) con una marcada desigualdad de ingresos en los hogares. La matriz de la desigualdad social también está condicionada por la cultura de privilegio, un rasgo histórico constitutivo de las sociedades de América Latina y el Caribe, que naturaliza las jerarquías sociales y las marcadas asimetrías de poder y de acceso a los activos productivos, y que sostiene un sistema de reglas, prácticas e instituciones que no garantizan la igualdad de oportunidades y de trato. La cultura de privilegios se basa en la negación del otro como sujeto de derechos y en esa medida instituye y reproduce privilegios económicos, políticos, sociales y culturales asociados a la condición étnico-racial, de género, origen, cultura, lengua y religión de las personas y grupos sociales ${ }^{5}$.

El primer eje de la matriz de la desigualdad sociales el estrato socioeconómico, cuyos elementos centrales incluyen la estructura de la propiedad y la distribución de los recursos y activos productivos y financieros. Una de sus manifestaciones más claras y evidentes es la desigualdad de ingresos, que es a su vez causa y efecto de otras disparidades en ámbitos como la salud, el acceso a los servicios básicos y la educación ${ }^{3}$. Pero también existen otros ejes que estructuran las desigualdades sociales en América Latina: el género, la condición étnica y racial, el territorio, la etapa del ciclo de vida, la situación de discapacidad, el estatus migratorio y la orientación sexual y la identidad de género (Cuadro 1). Lo que confiere a cada uno de estos ejes el carácter estructurante en la configuración de las desigualdades sociales es su peso constitutivo y determinante en el proceso de producción y reproducción de las relaciones sociales y de la experiencia subjetiva de las personas o, en otras palabras, su impacto sobre la magnitud y la reproducción de las desigualdades en distintos ámbitos del desarrollo y del ejercicio de los derechos ${ }^{3}$.

Los ejes estructurantes de la matriz de la desigualdad social se entrecruzan, potencian y encadenan a lo largo del ciclo de vida, lo que cimienta una multiplicidad de factores de desigualdad o discriminación que interactúan de forma simultánea y se acumulan a través del tiempo y de las generaciones ${ }^{3}$.

\section{Implicancias para la salud}

Existe evidencia incontrovertible sobre la relación entre los ejes de la matriz de la desigualdad social y las inequidades en salud. Desde los estudios seminales de Whitehall, se identificó una asociación inversa pronunciada entre la clase social, medida por la categoría ocupacional, y la mortalidad por una amplia gama de enfermedades 6 . Hay diversas evidencias que indican que el nivel de ingresos de los hogares influye en el estado de salud de sus miembros a través del consumo de alimentos saludables, la calidad de la vivienda, comportamientos de riesgo, acceso a servicios de salud de calidad y factores menos tangibles, como el capital social. Por otra parte, existen asociaciones directas entre la exposición permanente a la discriminación en base a raza y etnia y una amplia gama de trastornos mentales 
y condiciones físicas de salud 7 . Finalmente, y en línea con el concepto de la matriz de la desigualdad social, experimentar las múltiples y simultáneas formas de desigualdad asociadas a sus ejes estructurantes, que interactúan y se magnifican, da forma a experiencias de vida y realidades que derivan en inequidades en la salud que deben ser abordadas de manera integrada y holística.

Las desigualdades en salud, tanto en el acceso como en los resultados, no solo reflejan la vulneración del derecho a la salud, que además incide sobre el goce de otros derechos, sino que también son un eslabón central para la reproducción de la pobreza y la desigualdad, al reducir las capacidades y oportunidades en la esfera económica, obstaculizando la innovación y los aumentos de productividad. Las personas con buen estado de salud y nutrición presentan mejores capacidades físicas y mentales para el trabajo, así como tasas más bajas de ausentismo laboral. La salud también tiene un efecto indirecto sobre la productividad, al facilitar el desarrollo cognitivo, la capacidad de aprendizaje y el rendimiento escolar, así como la posibilidad de aprender y adquirir nuevas habilidades ${ }^{5}$. Por lo tanto, garantizar el derecho a la salud y reducir las brechas observadas en este ámbito son elementos fundamentales tanto para la erradicación de la pobreza y la reducción de la desigualdad como para el crecimiento económico y el desarrollo sostenible.

\section{Las desigualdades en salud: algunos indicadores}

En las últimas décadas se han registrado avances importantes en el estado de salud de la población de América Latina que hoy en día vive vidas más largas y sanas en comparación con períodos anteriores. Sin embargo, ese progreso ha sido desigual y millones de personas han sido dejadas atrás. Las desigualdades en salud se evidencian en marcadas diferencias en el acceso a servicios para la prevención, la detección y el tratamiento de condiciones de salud, segmentación en la calidad de dichos servicios, y en última instancia, en sus resultados. Considerando las herramientas, los recursos y los avances tecnológicos en el campo de la salud con los que contamos hoy, el buen estado de salud debe estar al alcance de todos y las desigualdades en salud no son aceptables.

El escenario de desigualdad que caracteriza a la región se expresa, por ejemplo, en las importantes desigualdades en salud que afectan a la niñez indígena y afrodescendiente. Esas desigualdades no solo representan una grave vulneración de sus derechos, sino que además tienen consecuencias para etapas posteriores del ciclo de vida ${ }^{8,9}$. La mortalidad infantil (la que ocurre antes del primer año de vida) es un indicador que refleja las desigualdades que afectan a los niños indígenas y afrodescendientes en América Latina desde el inicio de la vida (Gráfico 1). Para enfrentarlas es fundamental adoptar un enfoque intercultural en los sistemas de salud y de protección social y crear o fortalecer mecanismos de participación en la toma de decisiones de manera que las políticas y programas respondan de manera adecuada a las necesidades de estas poblaciones ${ }^{10}$.

Asimismo, se registran brechas importantes en otros indicadores de salud - por ejemplo, la esperanza de vida, indicadores de nutrición infantil, suicidio, salud materna, entre otros ${ }^{11}$. Además, existen disparidades notables en el acceso a servicios básicos de agua y saneamiento, que son claves para la salud y nutrición ${ }^{3}$, así como de afiliación a sistemas de salud según el estrato socioeconómico. En todos estos indicadores se evidencian disparidades asociadas a las múltiples dimensiones de la matriz de desigualdad social.

Aunque la afiliación o cotización a sistemas de salud ha aumentado desde comienzos de los años 2000 y las brechas socioeconómicas han disminuido, todavía hay un largo trecho por recorrer para alcanzar niveles de acceso más equitativos ${ }^{1}$. Como se muestra en el Gráfico 2, a pesar de un importante aumento en la cobertura, en especial en los primeros deciles de ingreso, lo que redujo las desigualdades entre los deciles, persiste una diferencia de 37 puntos porcentuales entre el decil de menores recursos y el de mayores recursos. Además, la afiliación o cotización no garantiza el acceso efectivo (que se puede ver restringido por barreras económicas, como co-pagos), ni la calidad de los servicios recibidos.

Estos desafíos reclaman la implementación de estrategias e intervenciones intersectoriales que aborden de manera holística las desigualdades en salud desde un enfoque de derechos. En particular es imperativo contar con sistemas de salud fortalecidos e integrados a sistemas de protección social universales y sensibles a las diferencias, que actúen de manera conjunta sobre los determinantes sociales de la salud -como la pobreza o la exclusión- a lo largo del ciclo de vida.

\section{Políticas de protección social para enfrentar las desigualdades en salud: reflexión y evidencias}

Según se destaca en el Informe de la Comisión de Alto Nivel "Salud Universal en el Siglo XXI: 40 años de Alma-Ata"10, sobre el cual se basa 
A. Población afrodescendiente y no afrodescendiente

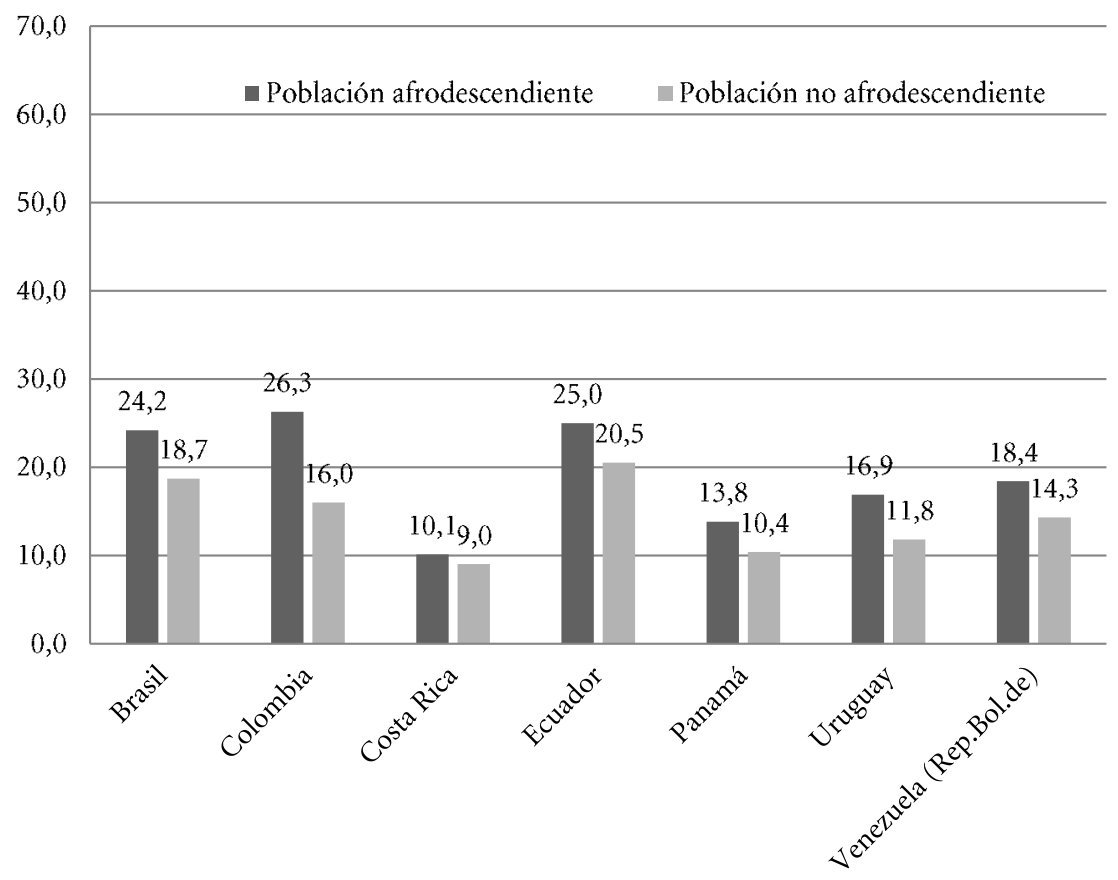

B. Población indígena y no indígena

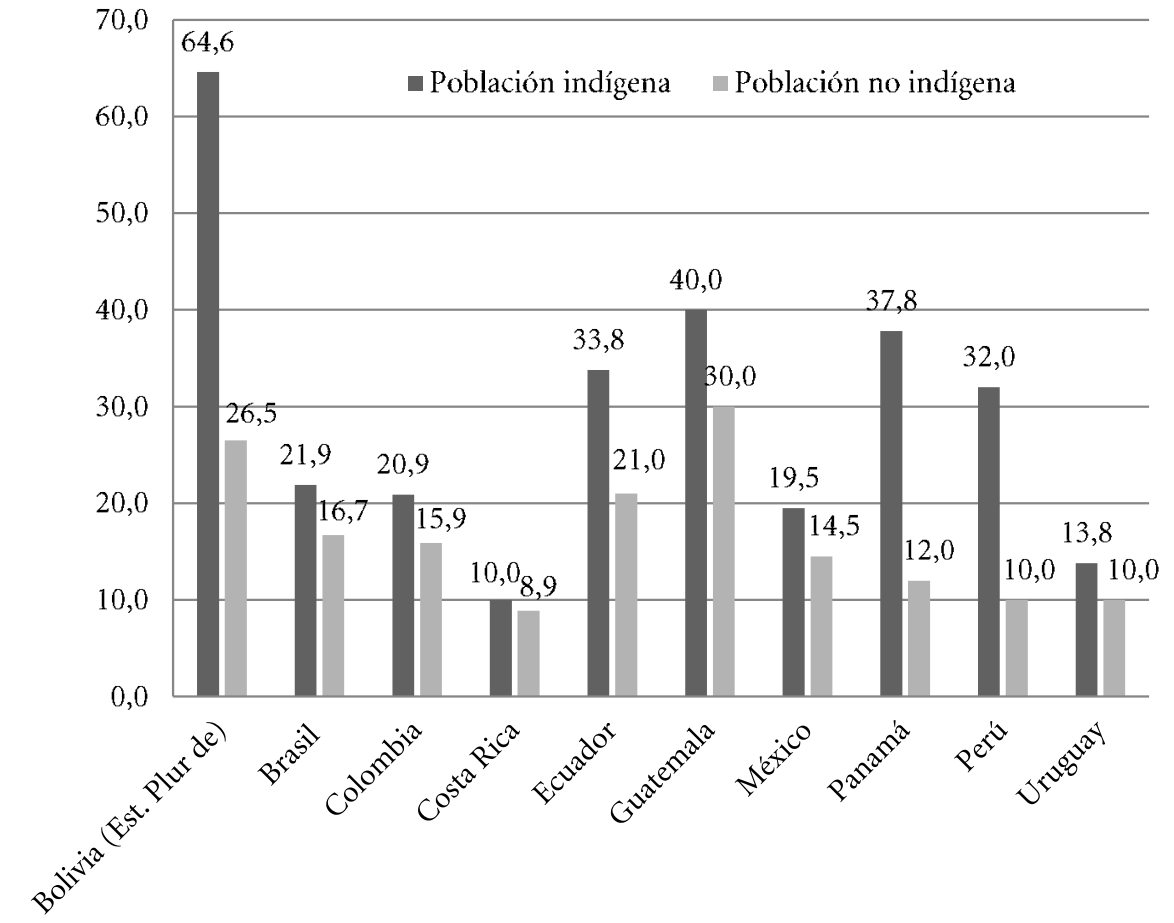

Gráfico 1. América Latina: mortalidad infantil en menores de 1 año de edad por raza y etnia alrededor de 2010. (Número de muertes por cada 1, 000 nacidos vivos).

Fuente: A: CEPAL (2017), Situación de las personas afrodescendientes en América Latina y desafíos de políticas para la garantía de sus derechos ${ }^{9}$ y B: CEPAL (2017), Brechas, ejes y desafíos en el vínculo entre lo social y lo productivo ${ }^{8}$. 


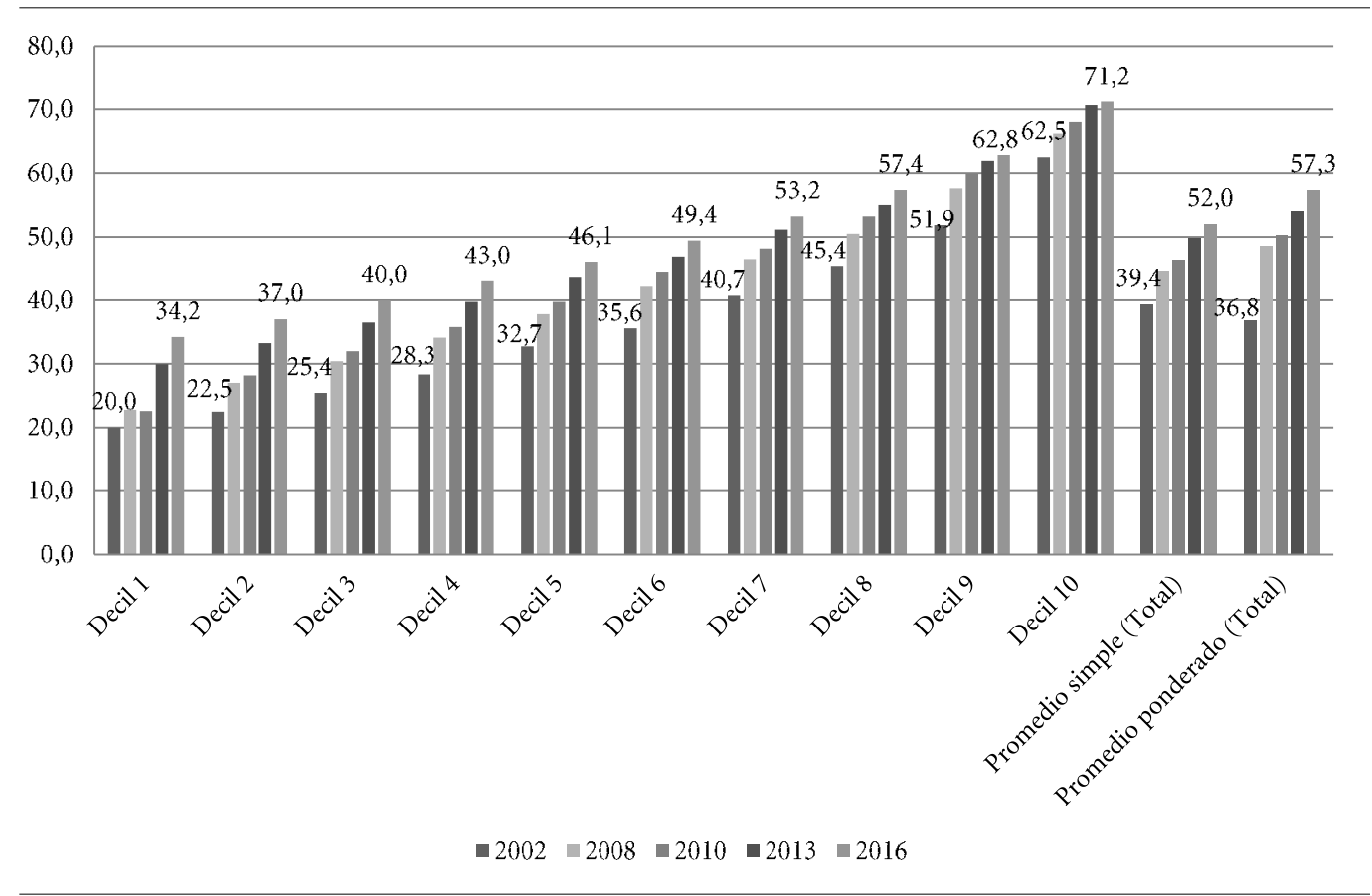

Gráfico 2. América Latina (14 países): afiliación o cotización a sistemas de salud de los ocupados de 15 años y más, según deciles de ingreso, totales nacionales, 2002-2016 /a /b. (En porcentajes).

Fuente: CEPAL, 2019 Panorama Social de América Latinal . a/ En la Argentina, corresponde a asalariados de 15 años y más. La información para México en 2016 no es estrictamente comparable con la de años anteriores debido a cambios en el fraseo de algunas de las preguntas relativas al acceso a la seguridad social. Véanse más detalles de estos cambios, sus efectos en la estimación de cobertura de seguridad social (salud y pensiones) y procedimientos para ajustar dicha estimación en CONEVAL (2017). b/ Promedio simple de: Argentina (zonas urbanas), Bolivia (Estado Plurinacional de), Chile, Colombia, Costa Rica, Ecuador, El Salvador, Guatemala, Honduras, México, Nicaragua, Paraguay, Perú y Uruguay (zonas urbanas).

en gran parte esta sección, existe un creciente reconocimiento sobre el rol de la protección social en la erradicación de la pobreza, la reducción de la vulnerabilidad y la desigualdad y en la promoción del desarrollo inclusivo, lo que tiene impactos positivos sobre la salud de la población.

La protección social se centra en tres ideas principales: garantías básicas de bienestar, aseguramientos frente a riesgos derivados del contexto o del ciclo de vida y moderación o reparación de daños sociales derivados de problemas o riesgos sociales. Siguiendo este concepto, la protección social está dirigida a responder no solo a los riesgos que enfrenta toda la población (por ejemplo, discapacidad o vejez), sino también a problemas estructurales, como la pobreza y la desigualdad ${ }^{12}$. Asimismo, se propone que la protección social sea entendida desde una visión amplia e integral, que comprende el conjunto de políticas y programas tanto de carácter no contributivo, como contributivo, tomando en cuenta las medidas de regulación del mercado laboral y los sistemas de cuidados ${ }^{13}$.

Los diversos mecanismos de protección social aminoran directamente los altos costos asociados a acudir a servicios de salud y mitigan el impacto de otros costos indirectos (como pérdidas de ingresos debido a enfermedad, discapacidad o desempleo, gastos no médicos asociados a utilizar servicios de salud (como transporte, alimentación, cuidados). De esta manera la protección social puede prevenir que los hogares caigan en la pobreza o que su situación de pobreza se profundice. Por otra parte, puede apoyar la superación de barreras de acceso experimentadas por poblaciones específicas, como afrodescendientes, indígenas, residentes en áreas rurales, entre otras. A continuación, se presentan algunos ejemplos de la interconexión entre mecanismos de protección social y la reducción de las desigualdades en salud. 


\section{La protección social en la infancia}

Desde una óptica de prevención, las primeras etapas del ciclo de vida son críticas: es durante estas etapas cuando se sientan las bases para el futuro desarrollo cognitivo, afectivo y social de las personas. Por lo tanto, intervenir en ellas puede contribuir a la reducción de las desigualdades en salud a largo plazo y frenar la transmisión intergeneracional de la pobreza y la desigualdad.

Una nutrición adecuada y sana desde edades tempranas y la adopción de buenos hábitos alimenticios puede contribuir a evitar problemas de salud en etapas posteriores del ciclo de vida. Existen diversas estrategias de protección social en el ámbito de la nutrición dirigidas principalmente a mujeres embarazadas, lactantes y preescolares, y a estudiantes de nivel primario y secundario. Entre estas se encuentran los programas de alimentación complementaria, alimentación escolar, fomento de la lactancia materna, distribución de alimentos y la suplementación y fortificación con micronutrientes. Algunos programas destacados son el Programa de Abasto Social de Leche de México, el Programa Nacional de Alimentação Escolar de Brasil, y el Programa Nacional de Alimentación Escolar Qali Warma de Perú.

Asimismo, las políticas de atención integral y los sistemas de protección integral a la primera infancia intentan articular el conjunto de acciones, políticas, planes y programas que ejecutan diferentes instancias del Estado junto con otros actores, en particular la sociedad civil, para garantizar que todos los niños y niñas gocen de sus derechos sin discriminación, al mismo tiempo que se atienden de forma focalizada las situaciones especiales ${ }^{8}$. Estas políticas buscan acompañar a los niños en su proceso de desarrollo a través de las diferentes etapas que componen la primera infancia, conjugando así intervenciones en salud, nutrición y educación temprana y cuidados. Ya que estas políticas están orientadas a proteger y promover los derechos de todos los niños y niñas, permiten además recuperar una visión de la política social dirigida a la infancia como algo que compete al conjunto de la sociedad y no algo que remite solo a los niños y niñas en situación de pobreza y pobreza extrema. Desde un enfoque multidimensional y con vías integradas de acción, estas políticas pueden crear un encadenamiento virtuoso de oportunidades y favorecer el desarrollo de capacidades a lo largo del ciclo de vida, reduciendo así las desigualdades $^{8}$.Algunos ejemplos de estas políticas son Brasil Carinhoso, Chile Crece Contigo, Uruguay Crece Contigo, De Cero a Siempre (Colombia) y Educa a tu Hijo (Cuba). Otros países que han avanzado sustantivamente en estrategias para la primera infancia son el Ecuador, Panamá, el Perú y la República Dominicana.

\section{Programas de transferencias condicionadas}

En las últimas dos décadas la mayoría de los países de América Latina han implementado programas de transferencias condicionadas (PTC) que están destinadas a familias que viven en condiciones de pobreza y pobreza extrema. Estos instrumentos de protección social no contributiva han tenido efectos positivos sobre diversos indicadores de salud y nutrición. Actualmente la región cuenta con 30 PTC en 20 países, alcanzando en 2017 a 133,5 millones de personas con un gasto equivalente al 0,37\% del PIB regional. Pese a compartir características comunes, son programas muy heterogéneos en cuanto a sus componentes, cobertura, montos transferidos, papel que cumplen y aplicación de las condicionalidades ${ }^{13-15}$.

Los PTC actúan a través de dos canales paralelos - por una parte buscan aumentar los recursos disponibles para el consumo de los hogares de bajos ingresos, con el fin de satisfacer sus necesidades básicas; por otra, fomentan el desarrollo humano de sus miembros con el fin de interrumpir la transmisión intergeneracional de la pobreza. Además, se facilita el acceso a una gama de servicios sociales a condición de que las familias participantes adhieran a ciertos compromisos en las áreas de educación, salud y nutrición.

La inclusión de condicionalidades de salud y de intervenciones de salud complementarias en los PTC (Cuadro 2) han servido para estimular la demanda de los servicios de salud, frecuentemente en áreas rurales remotas o urbanas marginales, donde su oferta escasea o es de calidad inferior. Por lo tanto, estos programas han tenido un efecto positivo, facilitando el acceso a los servicios de salud de grupos de la población tradicionalmente excluidos. Los PTC promueven la equidad, centrándose en identificar y abordar las mayores necesidades diferenciales de las personas en situación de pobreza. Además, pueden impulsar la cobertura universal de salud, adaptando los servicios a las necesidades de las personas socialmente excluidas e introduciendo un enfoque equitativo en los programas universales ${ }^{16}$.

Los PTC han sido objeto de numerosas evaluaciones ${ }^{15}$. La evidencia de los resultados de estos programas indica que hay una ampliación en el acceso a los servicios de salud de los niños, niñas y adolescentes de las familias participantes. Se han registrado también resultados positivos en diversos indicadores de salud y en la situación 
Cuadro 2. Condicionalidades de salud en los programas de transferencias monetarias condicionadas en América Latina, $2018^{\mathrm{a}}$.

\begin{tabular}{|c|c|c|c|c|c|c|c|}
\hline País & Programa & $\begin{array}{c}\text { Chequeos } \\
\text { médicos } \\
\text { (niños) }\end{array}$ & $\begin{array}{c}\text { Chequeos } \\
\text { médicos } \\
\text { (embarazadas) }\end{array}$ & $\begin{array}{c}\text { Chequeos } \\
\text { médicos } \\
\text { (adultos } \\
\text { mayores y } \\
\text { personas con } \\
\text { discapacidad) }\end{array}$ & $\begin{array}{l}\text { Vacunas } \\
\text { (niños) }\end{array}$ & $\begin{array}{c}\text { Vacunas } \\
\text { (embarazadas) }\end{array}$ & $\begin{array}{l}\text { Consejerías } \\
\text { en salud }\end{array}$ \\
\hline Argentina & $\begin{array}{l}\text { Asignación } \\
\text { Universal por } \\
\text { Hijo para } \\
\text { Protección } \\
\text { Social }\end{array}$ & 0-18 años & $\mathrm{X}$ & & $\begin{array}{l}0-18 \\
\text { años }\end{array}$ & $\mathrm{X}$ & $\mathrm{X}$ \\
\hline $\begin{array}{l}\text { Bolivia (Est. } \\
\text { Plur. de) }\end{array}$ & $\begin{array}{l}\text { Bono Madre } \\
\text { Niño-Niña } \\
\text { "Juana } \\
\text { Azurduy" }\end{array}$ & 0-2 años & $\mathrm{X}$ & & 0-2 años & $\mathrm{X}$ & $\mathrm{X}$ \\
\hline Brasil & Bolsa Família & 0-6 años & $\mathrm{X}$ & & 0-6 años & & \\
\hline Chile & $\begin{array}{l}\text { Seguridades y } \\
\text { Oportunidades } \\
\text { / Ingreso Ético } \\
\text { Familiar }\end{array}$ & 0-5 años & & & & & \\
\hline Colombia & $\begin{array}{l}\text { Más Familias en } \\
\text { Acción }\end{array}$ & 0-5 años & & & & & $\mathrm{X}$ \\
\hline Costa Rica & Avancemos & $\begin{array}{l}12-25 \\
\text { años }^{\text {b }}\end{array}$ & & & & & \\
\hline Ecuador & $\begin{array}{l}\text { Bono de } \\
\text { Desarrollo } \\
\text { Humano } \\
\end{array}$ & 0-5 años & $\mathrm{X}$ & & & & $\mathrm{X}$ \\
\hline El Salvador & $\begin{array}{l}\text { Programa } \\
\text { de Apoyo a } \\
\text { Comunidades } \\
\text { Solidarias en El } \\
\text { Salvador }\end{array}$ & 0-4 años & $\mathrm{X}$ & & 0-4 años & & \\
\hline Guatemala & Bono Social & 0-5 años & $\mathrm{X}$ & & & & \\
\hline Honduras & $\begin{array}{l}\text { Bono Vida } \\
\text { Mejor } \\
\end{array}$ & 0-5 años & $\mathrm{X}$ & & & & \\
\hline México & Prospera & 0-19 años & $\mathrm{X}$ & $\mathrm{X}$ & & & $\mathrm{X}$ \\
\hline Panamá & $\begin{array}{l}\text { Red de } \\
\text { Oportunidades }\end{array}$ & 0-4 años & $\mathrm{X}$ & & 0-4 años & & \\
\hline Paraguay & Tepokorâ & 0-18 años & $\mathrm{X}$ & $\mathrm{X}$ & $\begin{array}{l}0-18 \\
\text { años }\end{array}$ & & $\mathrm{X}$ \\
\hline Perú & Juntos & 0-5 años & $\mathrm{X}$ & & & & \\
\hline $\begin{array}{l}\text { República } \\
\text { Dominicana }\end{array}$ & $\begin{array}{l}\text { Progresando } \\
\text { con Solidaridad }\end{array}$ & 0-4 años & $\mathrm{X}$ & & & & $\mathrm{X}$ \\
\hline Uruguay & $\begin{array}{l}\text { Asignaciones } \\
\text { Familiares/Plan } \\
\text { de Equidad }\end{array}$ & 0-18 años & & $\mathrm{X}$ & & & \\
\hline $\begin{array}{l}\text { América } \\
\text { Latina }\end{array}$ & $\begin{array}{l}\text { Número de } \\
\text { programas con } \\
\text { condicionalidad } \\
\text { de salud }\end{array}$ & 16 & 11 & 3 & 6 & 2 & 7 \\
\hline
\end{tabular}

Fuente: Elaboración propia, sobre la base de Cecchini y Vera Soares ${ }^{16}$ y CEPAL, Base de datos de programas de protección social no contributiva en América Latina y el Caribe [en línea] https://dds.cepal.org/bpsnc.

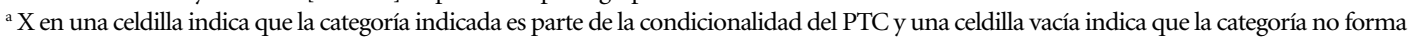
parte de las condicionalidades del programa. ${ }^{\mathrm{b}} \mathrm{La}$ Caja Costarricense de Seguro Social (CCSS) realiza una evaluación médica completa de los estudiantes matriculados en escuelas secundarias públicas durante el año escolar. 
nutricional ${ }^{17}$, aunque las evaluaciones indican cierta heterogeneidad de esos efectos positivos, dependiendo, por ejemplo, de la zona de residencia, sexo, edad y duración de la participación en el programa ${ }^{16}$. Desde luego las mejoras en los resultados de salud y nutrición asociados a estos programas dependen de la existencia de servicios en calidad y cantidad suficientes ${ }^{16}$. A nivel macro, un efecto positivo ha sido el aumento de la demanda de los servicios de salud, lo que ha estimulado la ampliación de la oferta de esos servicios y un mayor acercamiento del Estado y del abanico de políticas sectoriales y programas de promoción social a las poblaciones en situación de exclusión, reduciendo así las barreras de acceso y las desigualdades en salud.

\section{La universalización de la salud}

Si bien ha avanzado el debate sobre la universalización de la salud en la región y se ha ganado terreno sobre sus aspectos básicos, siguen existiendo desafíos conceptuales, prácticos y operacionales sobre cómo lograrla ${ }^{18}$. Durante la última década y media, distintas reformas de los sistemas de salud en América Latina, sostenidas por un aumento del gasto público del gobierno central en salud del 1,4\% del PIB en 2000 a 2,2\% en el $2016^{1}$, han permitido aumentar la cobertura y la equidad en el acceso. Si bien estas cifras no contemplan todo el gasto público social en salud, aún están muy distantes de lo recomendado por la Organización Panamericana de Salud (6\% del PIB). Además, las características de los sistemas de salud en términos de inversión, gasto de bolsillo, cobertura, resultados e integración entre los sistemas de salud pública y de seguridad social son muy diferentes entre los países. Estas diferencias están relacionadas a la evolución histórica del estado de bienestar, que a su vez está influenciada por factores económicos, sociales, demográficos y políticos propios de cada país.

En América Latina, Brasil (Sistema Único de Salud) y Cuba (Sistema Nacional de Salud) garantizan cobertura universal gratuita a la salud, financiada por impuestos generales, mientras que Costa Rica ha logrado la universalización mediante el seguro social, que a partir de la década de 1980 comenzó a incluir a los trabajadores informales y a las familias pobres ${ }^{19}$. Pero la cobertura universal no es suficiente para garantizar el acceso universal, donde todas y todos puedan hacer uso efectivo de los servicios de salud, sin enfrentar discriminaciones o barreras. Además, desde la mirada de la igualdad preocupa que persista una fuerte fragmentación y superposición de las prestaciones y de la cobertura, que se evidencian en las grandes disparidades en la calidad de los servicios a los que acceden distintos grupos de población. Generalmente, los sistemas de salud en América Latina se organizan alrededor de servicios del sector público para las personas en situación de pobreza, servicios del seguro social para los trabajadores formales y servicios privados para los que pueden pagarlos ${ }^{20}$. De esta manera estos sistemas se mantienen segregados y patentemente desiguales al ofrecer diferentes servicios y servicios de calidad distintos a diferentes grupos poblacionales, por lo que distan de ser sistemas verdaderamente universales y equitativos.

Las disparidades en el acceso y la calidad de los servicios de salud son una de las expresiones más claras de la desigualdad en la región. Para no dejar a nadie atrás, es necesario fortalecer el compromiso de los países con la cobertura y el acceso universal a la salud, un paso crucial para garantizar el derecho universal a la salud, así como para construir sistemas de protección social universales desde una perspectiva de derechos y avanzar en el cumplimiento de los compromisos consagrados en la Agenda 2030 para el Desarrollo Sostenible. También se debe avanzar para mejorar la calidad de los servicios de salud y promover un enfoque integral y holístico de la salud con el fin de crear relaciones sinérgicas positivas con otras dimensiones del bienestar. Existe amplia evidencia de que las políticas articuladas en materia de educación, mercado laboral, desarrollo local, protección social e igualdad de género y étnico-racial, entre otros, pueden contribuir a mejorar el estado de salud de la población.

Además, es importante tener en cuenta que, aun en países donde la ley garantiza la cobertura universal, el acceso efectivo a servicios de salud puede verse limitado por barreras económicas, geográficas, culturales, lingüísticas, de accesibilidad y actitudinales, entre otras. A la luz de esta situación, y de las múltiples desigualdades que caracterizan a las sociedades latinoamericanas, se deben adoptar políticas universales que sean sensibles a las diferencias. Estas políticas están basadas en un enfoque de derechos con un alcance universal, que aplican de manera complementaria mecanismos focalizados o de acción positiva para romper las barreras de acceso a los servicios de salud que enfrentan diferentes grupos de la población ${ }^{3,5,8}$. 


\section{La protección social y la Atención Primaria en Salud: estrategias complementarias que se afianzan mutuamente para avanzar hacia el pleno goce de los derechos}

La Atención Primaria en Salud (APS) es una estrategia centrada en las personas y en las comunidades que busca el ejercicio efectivo del derecho a la salud a través del acceso a servicios de salud esenciales integrados, de calidad y asequibles. A poco más de 40 años de la Declaración de Alma-Ata que instaló con fuerza a la APS en la agenda de salud global, persisten desafíos, lo que ha llevado a un llamado para revigorizar los esfuerzos en torno a la $\mathrm{APS}^{10}$.

Además de su rol central en la reducción de las desigualdades en salud, los instrumentos de protección social pueden fortalecer la APS para garantizar el derecho a la salud. Tal como se señala en la Declaración de Alma Ata, en el marco de los sistemas de salud, la APS cumple funciones que van más allá de ser el primer nivel de contacto de los individuos, la familia y la comunidad con el sistema nacional de salud e incluyen la provisión de servicios de promoción, prevención, tratamiento y rehabilitación. A su vez, los instrumentos de protección social pueden actuar sobre diversos frentes para fortalecer la APS y contribuir a garantizar a todas las personas un nivel de salud que les permita llevar una vida social y económicamente productiva, tal como se establece en la Declaración de Alma Ata. Mediante la expansión de la cobertura y el acceso universal, las acciones de promoción y prevención en salud y nutrición, el fortalecimiento de la coherencia de las políticas $y$, de manera muy fundamental, la lucha contra la pobreza, la desigualdad y la exclusión, la protección social juega un papel ineludible para el avance en la garantía del derecho a la salud en la región y la superación de desigualdades en esta área.

De esta manera la protección social y la APS deben ser concebidas como estrategias complementarias que se afianzan mutuamente para progresar hacia el pleno goce de los derechos, incluido el de la salud (Cuadro 3). Por ejemplo, en la medida en que los mecanismos de protección social están orientados a la reducción de los riesgos que enfrenta la población infantil, buscando garantizar una nutrición adecuada y el acceso a servicios de salud y de educación de calidad, pueden favorecer el sano desarrollo cognitivo, afectivo y social de niños y niñas, con efectos positivos para su salud y la reducción de las desigualdades en salud y en otros ámbitos, en esta y en las siguientes etapas de su ciclo de vida.

Por otra parte, los programas de transferencias condicionadas buscan ampliar el acceso de las familias participantes a los servicios locales de salud, así como promover una nutrición adecuada y proporcionar información y asesoría sobre temas de salud. Finalmente, en los países de la región se han llevado a cabo diversas reformas para ampliar la cobertura y la calidad de las prestaciones de instrumentos de protección social que contribuyen a promover la universalización de la salud y la reducción de las desigualdades ${ }^{21}$. Un ejemplo de ello es el Plan AUGE en Chile, en virtud de la cual se crearon las Garantías Explícitas en Salud (GES), que garantizan determinadas prestaciones relacionadas con la prevención, tratamiento y rehabilitación de enfermedades incluidas en un listado básico (entre ellas el VIH/ SIDA y distintos tipos de cáncer) ${ }^{22}$.

\section{Conclusiones}

La Agenda 2030 para el Desarrollo Sostenible expresa un consenso sobre la necesidad de avanzar hacia sociedades más inclusivas, solidarias y cohesionadas. A la vez, pone a las personas en el centro, promoviendo un modelo de desarrollo sostenible y haciendo un llamado a "no dejar a nadie atrás" en la senda del desarrollo y a atender primero a los más rezagados ${ }^{3}$. Por tanto, la Agenda 2030 tiene en su centro la preocupación con la reducción de la desigualdad entre los países y al interior de ellos, entendida desde un enfoque multidimensional y comprehensivo.

Combatir la desigualdad en todas sus expresiones es un imperativo ético. Pero en una región marcada por profundas brechas estructurales que se expresan en diversos ámbitos, incluido el de la salud, también es una condición necesaria para el desarrollo sostenible. Las desigualdades sociales no solo son un obstáculo clave para el goce efectivo de los derechos económicos, sociales y culturales; tienen, además, un impacto negativo en la productividad, la fiscalidad, la sostenibilidad ambiental y la mayor o menor penetración de la sociedad del conocimiento 5 .

Con una creciente desigualdad en el mundo (relacionada a crecientes niveles de concentración de la riqueza), incertidumbre geopolítica y económica, y un modelo de desarrollo que daña el medioambiente, la concreción de la Agenda 2030 enfrenta grandes desafíos. En América Latina esos desafíos son de alta magnitud, considerando la existencia de 184 millones de personas en situación de pobreza, de las cuales 62 millones en situación de extrema pobreza ${ }^{1}$, además de un porcentaje significativo en situación de vulnerabilidad a la pobreza. Eso, además de representar una violación de los derechos básicos a niveles 
Cuadro 3. Ejemplos de mecanismos de protección social que pueden reforzar la APS.

\begin{tabular}{|c|c|}
\hline $\begin{array}{l}\text { Componente de la } \\
\text { protección social }\end{array}$ & Intervenciones de protección social y su vínculo con elementos de la APS \\
\hline Pilar no contributivo & $\begin{array}{l}\text { Transferencias monetarias condicionadas: } \\
\text {. Ampliar el acceso a los servicios de salud, en particular los vinculados a la salud } \\
\text { materno-infantil, a poblaciones en situación de pobreza y de pobreza extrema } \\
\text {. Transmitir información sobre salud para la promoción y prevención a las } \\
\text { familias participantes } \\
\text {. Fomentar la coordinación intersectorial } \\
\text { Transferencias en especie (p. ej., programas de alimentación o suplementación } \\
\text { nutricional): } \\
\text { Atender las necesidades nutricionales de niños y niñas, en especial de los que viven en } \\
\text { situación de pobreza y pobreza extrema. Programas de atención integral a la primera } \\
\text { infancia: } \\
\text { - Articular acciones en salud, nutrición, educación y cuidados orientadas a la } \\
\text { primera infancia con miras a favorecer su desarrollo pleno } \\
\text {. Fomentar la coordinación intersectorial } \\
\text { Programas de promoción y acceso a la vivienda: } \\
\text { - Ampliar el acceso a viviendas que cuenten con servicios básicos y reducir la } \\
\text { exposición a riesgos sanitarios del entorno }\end{array}$ \\
\hline Pilar contributivo & $\begin{array}{l}\text { Aseguramiento de la salud: } \\
\text {. Expandir la cobertura y el acceso a servicios de salud } \\
\text {. Proporcionar protección financiera a los hogares } \\
\text { Licencias (de maternidad/paternidad y parentales) y protección contra el } \\
\text { despido durante el embarazo y el período post-parto: } \\
\text {. Proteger la salud física y mental de las trabajadoras durante el embarazo; facilitar } \\
\text { el apego de madres y padres y la lactancia materna }\end{array}$ \\
\hline
\end{tabular}

Fuente: Elaboración propia.

mínimos de bienestar (entre ellos el derecho a la salud) y protección social, significa también importantes límites al desarrollo del pleno potencial productivo y ciudadano de esas personas. Es una realidad injusta y que menoscaba las posibilidades de desarrollo sostenible en nuestra región.

Las profundas desigualdades en el estado de salud de las personas no pueden ser naturalizadas, como una dimensión más de la cultura del privilegio. El duro contraste entre la calidad de atención en el sistema público y el privado y las dramáticas disparidades en indicadores de salud tales como la mortalidad infantil, el embarazo adolescente no deseado y la esperanza de vida, entre otros, son una evidencia de la persistencia de privilegios en la sociedad y de distintos mecanismos de discriminación ${ }^{5}$. El acceso a la salud puede reducir brechas, siempre y cuando se trate de servicios de calidad, por lo que se requieren acciones concretas para, además de garantizar ese acceso a toda la población, equiparar la calidad de los servicios de salud.

Por otra parte, es importante recordar que los notables avances en el acceso y los resultados de salud de las últimas décadas ocurrieron un contexto económico favorable donde se priorizaron políticas orientadas a la reducción de la pobreza y la desigualdad, a la expansión y el fortalecimiento de sistemas de protección social y políticas activas en el mercado de trabajo. Aunque el contexto económico hoy en día es menos auspicioso, o precisamente debido a ello, ese tipo de políticas deben estar más presentes que nunca para resguardar los logros alcanzados y evitar retrocesos ${ }^{23}$.

Esta situación demanda respuestas integradas de políticas, capaces de crear sinergias entre diferentes sectores, haciendo uso eficiente de los limitados recursos disponibles, en búsqueda de objetivos comunes. Mantener una mirada amplia e integrada de la salud y la protección social, teniendo en cuenta la matriz de la desigualdad social y los determinantes sociales de la salud es clave para no dejar a nadie atrás en la senda del desarrollo en América Latina. La reducción de las desigualdades en salud debe ser una prioridad para todos los países del continente, independientemente de sus niveles de ingresos y de desarrollo. Un camino para avanzar en esa dirección es promover la construcción y el fortalecimiento de sistemas de protección social universales e integrados a lo largo del ciclo de vida en pro de la salud de la población y la reducción de las desigualdades en salud. 


\section{Colaboradores}

L Abramo, S Cecchini y H Ullmann contribuyeron a la elaboración, investigación y redacción de este texto.

\section{Referencias}

1. Comisión Económica para América Latina y el Caribe (CEPAL). Panorama Social de América Latina 2018. Santiago: Naciones Unidas; 2019.

2. Comisión Económica para América Latina y el Caribe (CEPAL). Coyuntura Laboral en América Latina y el Caribe. Santiago: Naciones Unidas; 2019. №20

3. Comisión Económica para América Latina y el Caribe (CEPAL). La ineficiencia de la desigualdad. Santiago: Naciones Unidas; 2019.

4. Comisión Económica para América Latina y el Caribe (CEPAL). Hacia una agenda regional de desarrollo social inclusivo: Bases y propuesta inicial. Santiago: $\mathrm{Na}-$ ciones Unidas; 2016.

5. Comisión Económica para América Latina y el Caribe (CEPAL). La matriz de la desigualdad social en América Latina. Santiago: Naciones Unidas; 2016.

6. Marmot MG, Shipley, MJ, Rose G. Whitehall I. Inequalities in death - specific explanations of a general pattern? Lancet 1984; 1(8384):1003-1006.

7. Lewis T. Self-reported experiences of discrimination and health: scientific advances, ongoing controversies, and emerging issues. Annu Rev Clin Psychol 2017; 11:407-440.

8. Comisión Económica para América Latina y el Caribe (CEPAL). Brechas, ejes y desafíos en el vínculo entre lo social y lo productivo. Santiago: Naciones Unidas; 2017.

9. Comisión Económica para América Latina y el Caribe (CEPAL). Situación de las personas afrodescendientes en América Latina y desafíos de políticas para la garantía de sus derechos. Santiago: Naciones Unidas; 2017.

10. Organización Panamericana de la Salud (OPS). Salud Universal en el Siglo XXI: 40 años de Alma-Ata: Informe de la Comisión de Alto Nivel. Washington DC: OPS; 2019.

11. Organización Panamericana de la Salud (OPS). Sociedades justas: equidad en la salud y vida digna. Resumen Ejecutivo del Informe de la Comisión de la Organización Panamericana de la Salud sobre Equidad y Desigualdades en Salud en las Américas. Washington: OPS; 2018.

12. Cecchini S, Filgueira F, Martínez R, Rossel C. Instrumentos de protección social: caminos latinoamericanos hacia la universalización. Santiago: Naciones Unidas; 2015. [Libros de la CEPAL no 136]

13. Cecchini S, Martínez R. Protección social inclusiva en América Latina: una mirada integral, un enfoque de derechos. Santiago: Naciones Unidas; 2011. [Libros de la CEPAL no 111]
14. Cecchini S, Atuesta B. Programas de transferencias condicionadas en América Latina y el Caribe: tendencias de cobertura e inversión. Santiago: CEPAL; 2017. [Serie Políticas Sociales, no 224 (LC/TS.2017/40)]

15. Abramo L, Cecchini S, Morales B. Programas sociales, superación de la pobreza e inclusión laboral. Aprendizajes desde América Latina y el Caribe. Santiago: Naciones Unidas; 2019. [Libros de la CEPAL no 155]

16. Cecchini S, Veras Soares F. Conditional cash transfers and health in Latin America. Lancet 2014; 385(9975):e32-e34.

17. Owusu-Addo E, Cross R. The impact of conditional cash transfers on child health in low- and middle-income countries: a systematic review. Int J Public Health 2014; 59(4):609-618.

18. Horton R, Das P. Cobertura universal en salud: no por qué, qué, ni cuándo, sino ¿cómo?. MEDICC Review 2015; 17(S1):S3-4.

19. Acosta OL, Cecchini S. Latin American pathways to achieve universal health coverage, en Health policy in emerging economies: innovations and challenges. Policy in Focus 2016; 3(1):7-9.

20. Titelman D, Cetrángolo O, Acosta OL. La cobertura universal de salud en los países de América Latina: cómo mejorar los esquemas basados en la solidaridad. MEDICC Review 2015; 17(S1):S68-S72.

21. Organización Mundial de la Salud (OMS). Estrategia para el acceso universal a la salud y la cobertura universal de salud. Ginebra: OMS; 2014.

22. Rossel C, Filgueira F. Etapa activa y reproductiva. En: Cecchini S, Filgueira F, Martínez R, Rossel C, organizadores. Instrumentos de protección social: Caminos latinoamericanos hacia la universalización. Santiago: Naciones Unidas; 2015. (Libros de la CEPAL nº 136). p. 171-224.

23. Rasella D, Basu S, Hone T, Paes-Sousa R, Ocké-Reis CO, Millett C. Child morbidity and mortality associated with alternative policy responses to the economic crisis in Brazil: A nationwide microsimulation study. PLoS Med 2018; 15(5):e1002570.

Artículo presentado en 30/05/2019

Aprobado en 07/08/2019

Version final presentada en 08/11/2019 